\title{
Effects of a Lateral Confinement on FC-72 Pool Boiling from a Plane Surface
}

\section{Mario Misale* \\ Department of Mechanics, Energetics, Management and Transportation, University of Genoa, Italy}

*Corresponding author: Mario Misale, Department of Mechanics, Energetics, Management and Transportation, University of Genoa, Via Opera Pia 15-a, (I) 16145, Genova, Italy, Tel: 39-0103532576; Fax: 39-010311870; E-mail: mario.misale@unige.it

Rec date: Apr 20, 2016, Acc date: May 12, 2016, Pub date: May 16, 2016

Copyright: (C) 2016 Misale M. This is an open-access article distributed under the terms of the Creative Commons Attribution License, which permits unrestricted use, distribution, and reproduction in any medium, provided the original author and source are credited.

\begin{abstract}
An experimental study was carried out on pool boiling from a plane surface. The influences of two different periphery conditions (pool boiling surface confinement) were investigated. One condition involved the use of a 200 $\mathrm{mm}$ diameter glass cylinder (single confinement); the other condition was achieved by inserting a $70 \mathrm{~mm}$ diameter cylinder inside the first one (double confinement). The experiments were performed at atmospheric pressure in a saturated pool of FC-72 (dielectric fluid). The experimental data were compared with those obtained in the case of an unconfined pool boiling surface. The presence of the confinement seems to improve heat transfer performance at low and moderate heat fluxes, whereas it has no effect at the maximum heat flux. For the latter situation, the images taken during the experiments show different flow patterns on the boiling surface.
\end{abstract}

Keywords: Pool boiling experiments; Periphery conditions; Dielectric fluid; Bubble visualization

\section{Introduction}

Improvements in cooling technologies are continually requested in many engineering applications, such as the cooling of electronic devices [1-3]. In additional to the usual air-cooling and indirect liquid cooling technologies, direct liquid immersion cooling soon proved to be very promising, especially where high thermal fluxes had to be transferred while keeping temperature increments of the electronic components to a minimum. In particular, many studies investigated pool boiling as the heat transfer mechanism; indeed, unlike singlephase forced convection, falling film, and flow boiling, pool boiling does not need a mechanical pump. Pool boiling cooling technology is therefore less complex, free from noise and pump-induced vibrations, and more reliable.

In many cases, different techniques can be used to ensure high performance of the boiling system: extended surfaces [4] and structured porous coated surfaces [5]. A possible alternative involves confinement of the boiling surface: an unheated wall which can induce different bubble flow patterns able to increase the pool boiling performance is placed in front of the boiling surface. In particular, two different confinement conditions have been considered, the first adopting open periphery conditions (the lateral sides of the channel formed by the unheated wall are open), the second one adopting closed periphery condition (all sides of the channel are closed except the top side and/or the bottom side).

The presence of an unheated wall in front of the boiling surface, especially fluxes, causes a significant increase $(300 \%-800 \%)$ in pool boiling performance [6-8], as compared with unconfined boiling, whereas it causes a drastic reduction in pool boiling performance at high fluxes.

This paper reports an experimental study on the pool boiling of a dielectric fluid (FC-72) on a plane surface in different periphery conditions. The boiling surface was confined, on the lateral side, by means of different glass cylinders. Moreover, to better understand the boiling performance, a CCD camera was used to observe the influence of confinement on the bubble flow patterns.

\section{Experiments}

\section{Experimental apparatus}

The experimental apparatus (Figure 1) utilized in the tests consists of an airtight stainless-steel vessel (380mm I.D. and $450 \mathrm{~mm}$ height) containing the test section assembly immersed in a pool of a saturated dielectric fluid (Fluorinert FC-72). An auxiliary heater controlled by a PID regulation system is wrapped around the vessel to pre-heat the liquid and to compensate for heat loss. At high heat fluxes, a watercooler condenser placed in the vapor space maintains saturation conditions.

The heat source module (Figure 2) consists of a copper block (99.9\%), used as a heat flux meter, which is thermally insulated by alumina) washers and heated on the lower side by a flat heater. The boiling area is the circular upper surface of the flux meter, which has a diameter of $40 \mathrm{~mm}$, corresponding to a base area, $\mathrm{Ab}$, of $12.56 \cdot 10^{-4} \mathrm{~m}^{2}$.

The surface temperature and the heat flux through the copper test section were calculated on the basis of the temperatures measured by nine calibrated $\mathrm{K}$-type thermocouples lodged inside the copper flux meter at different depths, and located at three planes $26 \mathrm{~mm}$ apart, starting $3 \mathrm{~mm}$ below the boiling surface. Liquid and vapor temperatures were measured by four calibrated K-type thermocouples, three of them immersed in the liquid at different depths. All temperatures were recorded by means of a high-precision dataacquisition system (Keithley 705 scanner, Keithley 196 DMM, electronic ice point ICELL MK). The chain of instruments used to detect the signals from the thermocouples yields an accuracy of \pm 0.1 $\mathrm{K}$. Considering the heat conduction through the test section, the maximum error associated for $10^{4}$ the heat flux in the region of 
Page 2 of 5

nucleate boiling was less than $5 \%$, while the maximum uncertainty associated with the surface temperature was estimated to be less than \pm $0.2 \mathrm{~K}$. Further details of the apparatus are reported in Ferrando et al. [9].

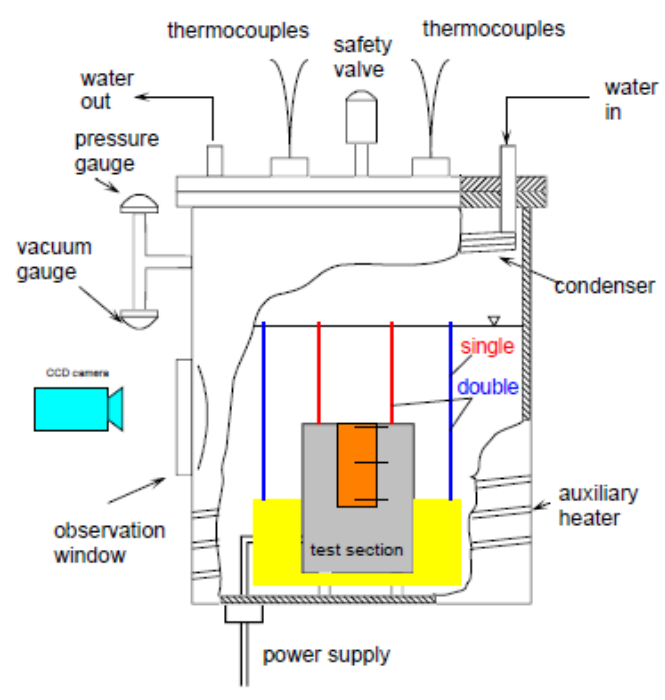

Figure 1: Experimental apparatus and different types of confinements.

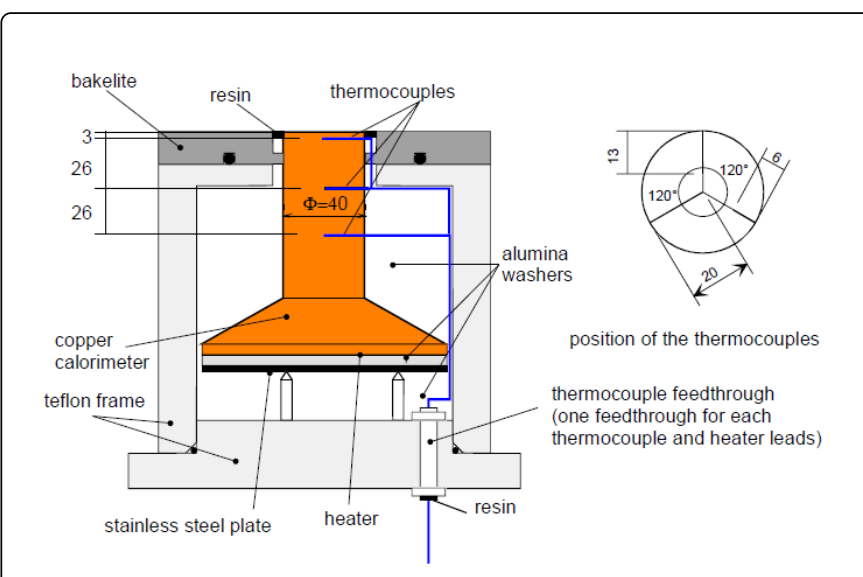

Figure 2: Test section.

The experiments were performed at atmospheric pressure. During the runs, several pictures were taken by means of a CCD camera. The pictures taken at the same heat flux were compared for the three periphery conditions investigated: unconfined (the test section was completely immersed in the FC-72 pool), single confinement (a glass cylinder of $200 \mathrm{~mm}$ was placed between the vessel wall and the test section) double confinement (a glass cylinder of $70 \mathrm{~mm}$ was placed between the previous glass cylinder and the test section).

\section{Test surface and experimental procedure}

As is well known, pool boiling performance depends on several parameters: fluid characteristics, saturation pressure, roughness of the boiling surface, and so on. With regard to the roughness of the boiling surface, it was considered advisable to use a sandblasting technique with controlled grade particles, after carrying out a series of preliminary tests, in order to obtain a sufficiently uniform and reproducible surface condition. This procedure was adopted to realize the test surface. Surface roughness was measured in several directions and the average $\mathrm{Ra}$ value was $0.76 \mu \mathrm{m}$. The standard deviation associated was $0.01 \mu \mathrm{m}$.

The heat flux q" transmitted to the liquid was determined on the basis of the average temperatures at the three different levels inside the copper calorimeter. The boiling surface temperature was obtained by extrapolation from the average temperature at the top of the three levels.

All the experiments were conducted in steady-state conditions. The following operating procedure was adopted: before filling the vessel, it was evacuated by means of a vacuum pump. When the vessel pressure decreased to $1.0 \mathrm{~Pa}$, the vessel was filled with FC-72. A degassing operation was then carried out until correspondence between pressure and saturation temperature was obtained. During the runs, the following procedure was adopted: before each test, the heating surface was left immersed in the saturated liquid overnight, with nil heat flux; the run was carried out by supplying the electric heater of the test section with gradually increasing power by modifying the voltage applied to the heater. When heat flux conditions close to the critical value were achieved, the voltage was reduced in steps of the same magnitude. For each power level set, steady-state conditions were attained in about 45 minutes for low heat fluxes and 15 minutes with fully developed boiling.

\section{Results and Discussion}

\section{Pool boiling data}

The experiments were conducted using a copper heat flux meter, confined by means different glass cylinders.

Several papers available in literature $[6,10]$ shows the presence of a confining wall causes an increase in the transfer performance at low and moderate heat fluxes, whereas at heat flux values close to the CHF, there is a drastic reduction in heat transfer performance. This reduction is caused by the difficulty for the vapor to escape and, at the same time, for the liquid to have the possibility to rewet the boiling surface. In the present study, however, the confinement is different: in front of the boiling surface, there is no wall to influence the vapor escape; rather, the confining walls are placed at the periphery of the boiling surface.

The boiling curves (q" vs. $\Delta$ Tsat) were obtained by varying the heat flux when the saturation pressure was 1.0 bar. Figure 3 shows the boiling data for the different confinement conditions; in the same figure, the boiling data for the unconfined condition are also reported. In Figure 3, full symbols indicate the increasing power phase, whereas the decreasing phase is indicated by empty symbols. It will be observed that some symbols are larger than others; these symbols have a cross and are edged in yellow. For these boiling data, several pictures taken during the experiments will be shown below.

The boiling characteristics show a hysteresis effect, owing to which the increasing power curve is separated from the decreasing curve. In particular, the boiling curves showed no difference only when departure from nucleate boiling was achieved. The effect of 
confinement is more evident at low and moderate heat fluxes; when departure from nucleate boiling is achieved, the amount of vapor produced and the bubble flow patterns do not seem to influence boiling performance. The increase in heat transfer performance is more evident for the experiments conducted in double confinement conditions. Moreover, the hysteresis effect is less pronounced in the case of double confinement. The reduction in the volume occupied by the liquid and the vapor produced probably increases the possibility of activating the nucleation sites on the boiling surface.

In Figure 4 the same data depicted in Figure 3 are re-arranged to plot the heat transfer coefficient versus the wall superheat. As can be seen, the heat transfer coefficient is influenced by the different kinds of confinement. The amount of the increase in the heat transfer coefficient depends on whether the data are compared in the case of increasing or decreasing heat flux. For example, at the wall superheat of $8 \mathrm{~K}$, the heat transfer coefficient for the single confinement condition is $130 \%$ higher than in the unconfined situation, while in the case of double confinement the increment of the heat transfer coefficient is $167 \%$. The hysteresis effect significantly influences the heat transfer coefficient. Considering the same wall superheat, comparison of the heat transfer coefficients measured on increasing or decreasing the heat flux shows that, for a wall superheat of $9 \mathrm{~K}$, their increments are almost the same: $340 \%, 360 \%$ and $360 \%$ for unconfined, single confinement and double confinement, respectively. By contrast, for the wall superheat of $10 \mathrm{~K}$, the corresponding increments are $630 \%, 320 \%$ and $130 \%$, respectively. This reduction is probably because the double confinement, as reported above, allows better insemination of the nucleation sites, thereby reducing the influence of the operating procedure adopted.

Figures 3 and 4 show that the slopes of the boiling curves change and that the slope decreases above $15 \mathrm{~K}$ for $\Delta$ Tsat. This slope change coincides with the beginning of the reduction in heat transfer coefficient values $\mathrm{h}$.

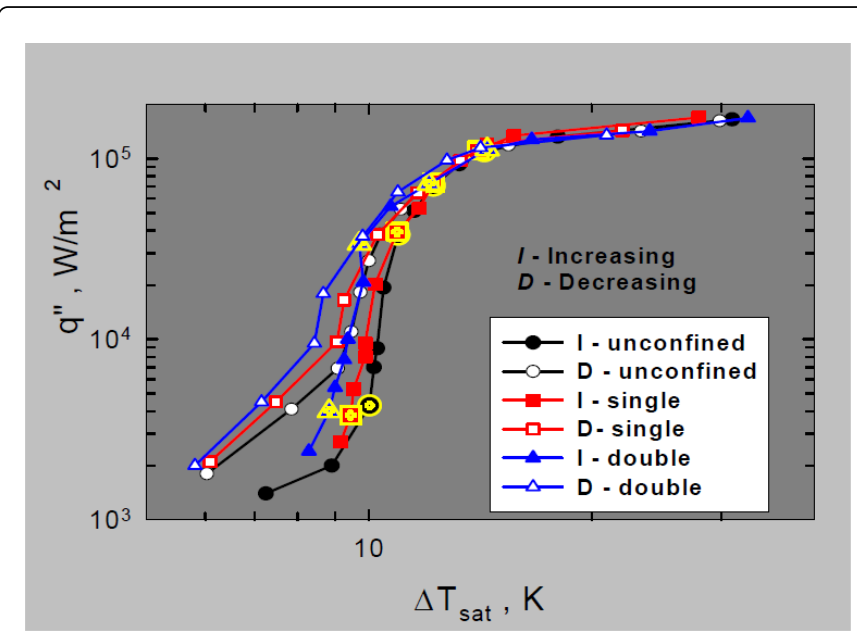

Figure 3: Effect of periphery conditions on pool boiling performance.

The wall superheat value of $15 \mathrm{~K}$ is the point at which the boiling regime deviates from nucleate boiling and is not influenced by the type of confinement. This regime displays a reduction in heat transfer coefficient for wall superheat values above $15 \mathrm{~K}$. The trend towards a maximum can be explained by the fact that boiling intensifies as the wall temperature rises. This trend is independent of the kind of confinement, as will be clearly seen in the following section, in which some boiling pictures are shown.

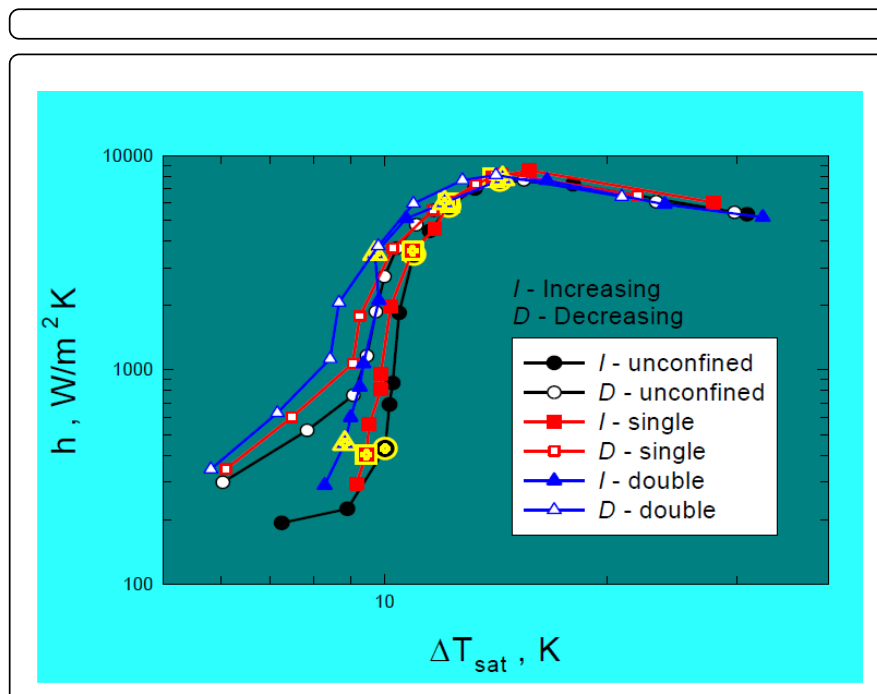

Figure 4: Heat transfer coefficient vs. wall superheat for different periphery conditions. Bubble visualization.

As reported above, the presence of lateral confinement leads to an increase in the pool boiling performance, especially at low and moderate heat fluxes, whereas it has no influence at high heat fluxes. These results are completely different from those reported in the literature quoted above. In those studies, the confinement was placed in front of the boiling surface, whereas in this paper the confinement is situated at the periphery of the boiling surface.

On analyzing the boiling data depicted in Figures 3 and 4, the above conclusions can be drawn. However, to better understand the boiling phenomena, further investigations should be performed. These inquiries can be carried out by using: a high-speed camera (1000 frames/s) [11], thermochromic liquid crystal [12], a hot-wire anemometry technique for phase -detection [13], and single photography by laser-Doppler anemometry [14]. The most useful technique is probably that of the high-speed camera, as it yields boiling information frame by frame, independently of the heat flux applied, which can determine different amounts of vapor production. Indeed, the visual technique in some cases is not applicable at high heat fluxes, when the amount of vapor produced and the bubble flow pattern involve interaction among the bubbles.

In this study, the heat flux transferred to the saturated fluid and the wall superheat were measured during the pool boiling experiments; moreover, at the same time, some moving pictures were taken with a CCD camera. In particular, almost the same heat flux was dissipated for the different confinement conditions. Working in this way, it was able to observe the possible different bubble flow patterns.

Some images were obtained from the moving pictures; these are shown in Figures 5-8. Each image consists of three pictures, one for each type of confinement, at four values of the heat flux transferred to the fluid (Figure 5, q" $=0.40 \cdot 10^{4} \mathrm{~W} / \mathrm{m}^{2}$; Figure 6, q" $=3.9 \cdot 10^{4} \mathrm{~W} / \mathrm{m}^{2}$; Figure $7, \mathrm{q}^{\prime \prime}=7.3 \cdot 10^{4} \mathrm{~W} / \mathrm{m}^{2}$; Figure $8, \mathrm{q}^{\prime \prime}=11.1 \cdot 10^{4} \mathrm{~W} / \mathrm{m}^{2}$ ). Moreover, Figures $5 c$ and $8 c$ show how the double confinement was realized. The boiling coordinates (q", $\Delta$ Tsat) of the data shown in the pictures can be 
Citation: Misale M (2016) Effects of a Lateral Confinement on FC-72 Pool Boiling from a Plane Surface. J Fundam Renewable Energy App 6:

characterized by the larger symbols in Figures 3 and 4 . At the lowest heat flux (Figure 5) it will be seen that, independently of the type of confinement, boiling started on a small patch, typically at the edge of the boiling surface. As heat flux increased, the boiling front expanded until the entire surface was undergoing nucleate boiling (Figure 6). As reported above, the boiling performance of the double confinement is better than others, especially at low and moderate heat fluxes. As can be observed on comparing Figures 5 and 6, it seems that the amount of vapor produced is lower in the case of the double confinement; however, the presence of the double confinement causes an increase in the heat transfer performance. Probably, to justify these results, a highspeed camera should be used. Indeed, this might well reveal whether the presence of the double confinement affects the number of nucleation sites as well as the frequency of bubble formation. Comparison between Figures $5 \mathrm{c}$ and $6 \mathrm{c}$ is more interesting: at $\mathrm{q}^{\prime \prime}=$ $0.40 \cdot 10^{4} \mathrm{~W} / \mathrm{m}^{2}$ a vortex appears on the boiling surface; as the heat flux increases, the vortexes grow and an interaction may take place among them. At low and moderate heat fluxes, the appearance of these vortexes and their movement on the boiling surface might accelerate the insemination of nucleation sites, thereby reducing the wall superheat for the same heat flux. This notion is confirmed by the fact that the heat flux has to be reduced in order to eliminate the difference between the boiling data during increasing and decreasing heat fluxes. When the departure from nucleate boiling occurs (Figures 7 and 8; q" $=7.3 \cdot 10^{4} \mathrm{~W} / \mathrm{m}^{2}$; Figure $8, \mathrm{q}^{\prime \prime}=11.1 \cdot 10^{4} \mathrm{~W} / \mathrm{m}^{2}$, respectively), the presence of vortexes on the boiling surface becomes more evident (Figures $7 \mathrm{c}$ and $8 \mathrm{c}$ ), whereas the bubble flow patterns for unconfined or single confinement seem more similar. At the former heat flux, bubble occurs, while at the latter heat flux the boiling regime is characterized by the formation of vapor mushrooms. However, it should be noted that, even though the bubble flow patterns are different, the presence of the confinement does not have any influence on boiling performance after the departure of nucleate boiling.
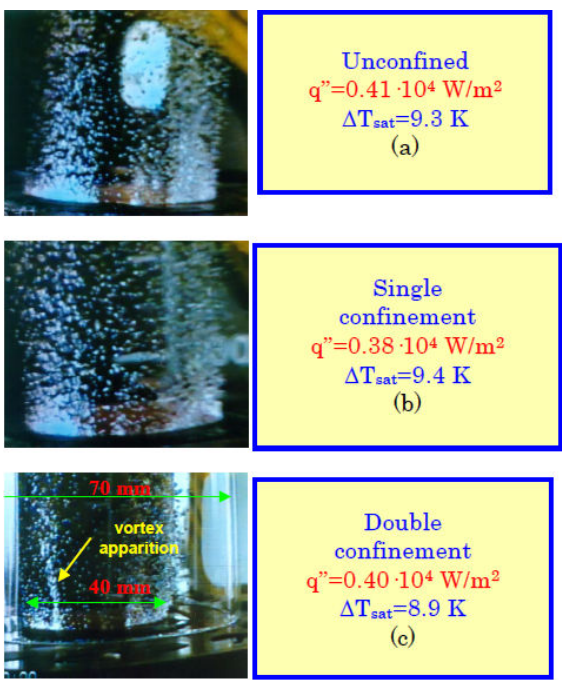

Figure 5: Visualization of the bubbles flow patterns for different periphery conditions: (a) Unconfined (b) Single confinement (c) Double confinement.
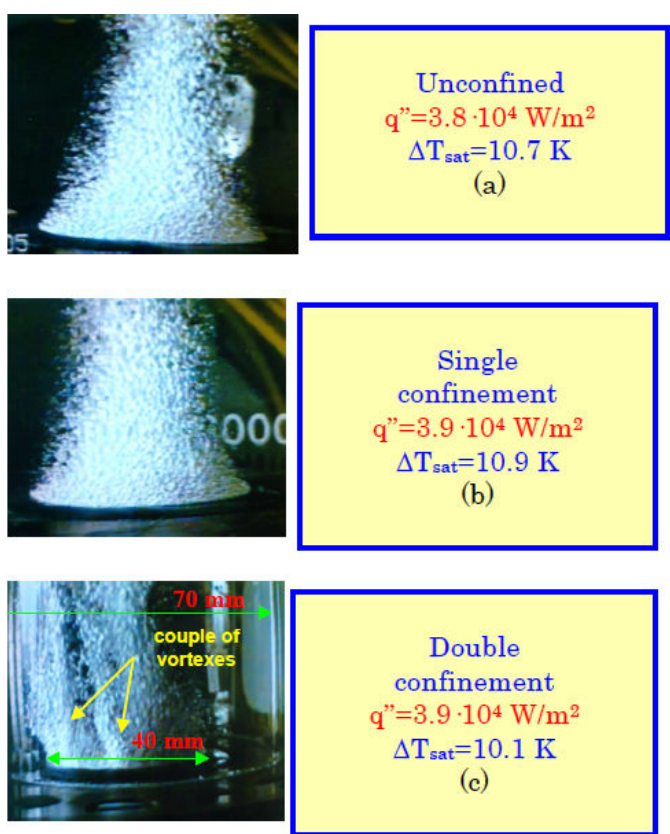

Double confinement q" $=3.9 \cdot 10^{4} \mathrm{~W} / \mathrm{m}^{2}$

$\Delta \mathrm{T}_{\text {sat }}=10.1 \mathrm{~K}$

(c)

Figure 6: Visualization of the bubbles flow patterns for different periphery conditions: (a) Unconfined (b) Single confinement (c) Double confinement.
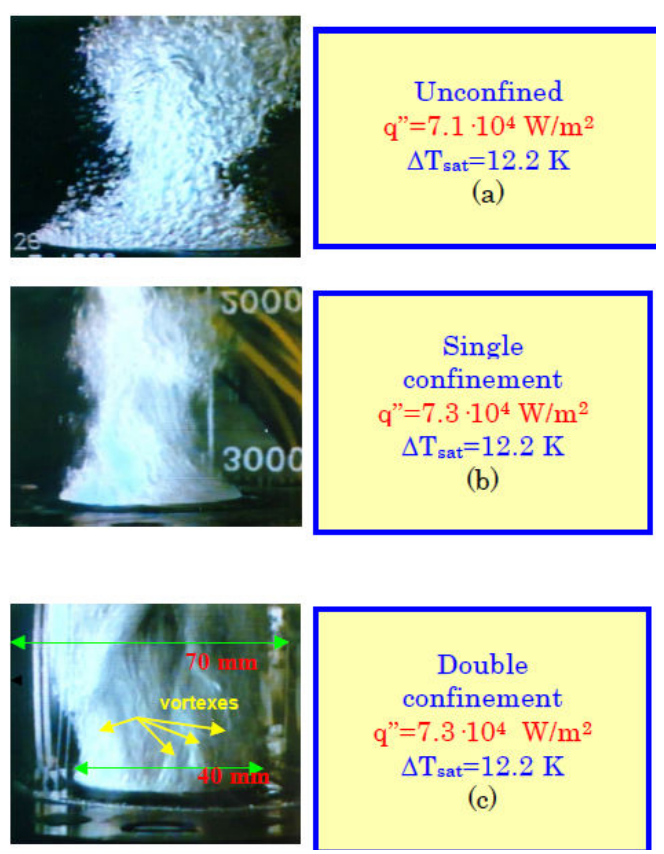

Figure 7: Visualization of the bubbles flow patterns for different periphery conditions: (a) Unconfined (b) Single confinement (c) Double confinement. 

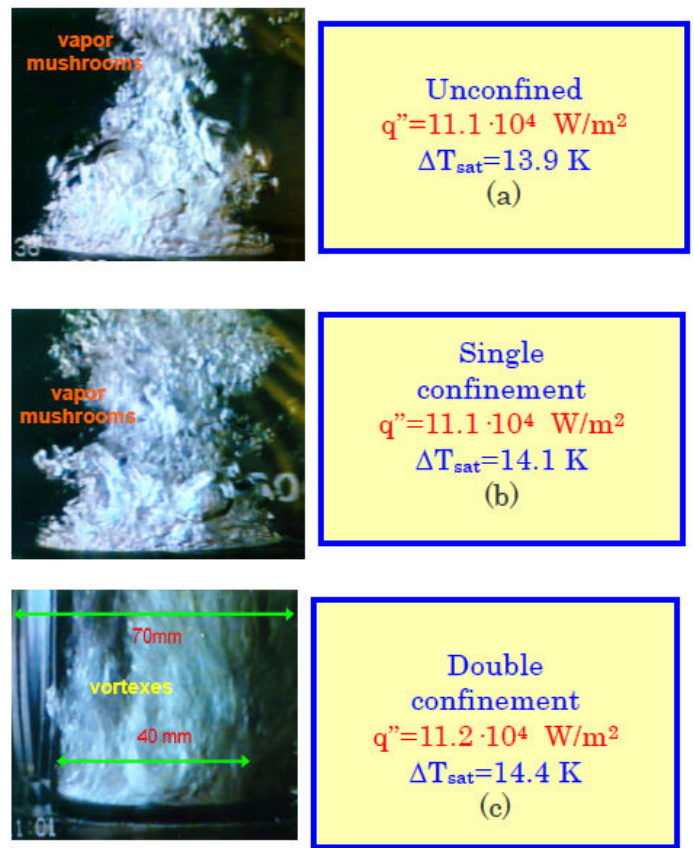

Figure 8: Visualization of the bubbles flow patterns for different periphery conditions: (a) Unconfined (b) single confinement (c) double confinement.

\section{Conclusions}

The boiling behaviour of a flat surface in a saturated pool of highly wetting fluid (FC-72) was analyzed. In particular, the effect of confinement (different periphery conditions) was examined. The experiments were performed by considering three periphery conditions: unconfined (the test section was completely immersed in the FC-72 pool), single confinement (a glass cylinder of $200 \mathrm{~mm}$ was placed between the vessel wall and the test section) double confinement (a glass cylinder of $70 \mathrm{~mm}$ was placed between the previous glass cylinder and the test section).

Confinement seems to improve heat transfer performance at low and moderate heat fluxes, whereas it has no effect at the maximum heat flux. In particular, the boiling data show a hysteresis effect (different boiling curves on increasing or decreasing the heat flux); however, double confinement yields better heat transfer performance, and the hysteresis effect is less pronounced.

The images taken during the experiments show different bubble flow patterns on the boiling surface. For the unconfined and single confinement conditions, the bubble flow patterns observed are quite similar; after the establishment of patch boiling, the surface is completely covered by bubbles, which, at high heat fluxes, induces the appearance of mushroom bubbles. A different behaviour was observed in the case of double confinement: few vortexes appeared on the boiling surface at low heat flux; as the heat flux increased, the number of vortexes increased and their movement on the surface gave rise to their interaction.

\section{References}

1. Bergles AE (1987) Liquid cooling for electronic equipment, International Symposium on Cooling Technology for Electronic Equipment, Honolulu, Hawaii.

2. Incropera FP (1990) Liquid cooling of electronic components, Heat Transfer in Electronic and Microelctronic Equipment. Elsevier Publ Corp Washington D.C 407-444.

3. Bergles AE, Bar-Cohen A (1994) Cooling of electronic systems, Kluwer Academic Publishers.

4. Mudawar I, Anderson TM (1993) Optimization of Enhanced Surfaces for High Flux Chip Cooling by Pool Boiling. J Electron Packag 115: 89-100.

5. Pais C, Webb RL (1991) Literature Survey of Pool Boiling on Enhanced Surfaces. ASHRAE Transaction Res 97: 79-89.

6. Bonjour J, Lallemand M (1995) Influence de la pression et du confinement sur les thermiques au cours de l'ébullition convective naturelle. Revue Générale Thermique 34: 667-677.

7. Kim JH, Rainey KN, You SM, Pak JY (2002) Mechanism of nucleate boiling heat transfer enhancement from microporous surfaces in saturated FC-72. J Heat Transfer 124: 500-506.

8. Yao SC, Chang Y (1983) Pool boiling heat transfer in a confined space. Int J Heat and Mass Transfer 26: 841-848.

9. Ferrando F, Guglielmini G, Misale M, Schenone C (1991) Una apparecchiatura sperimentale per lo studio dellebollizione nucleata di fluidi dielttrici. Energy Engineering Department, EGR/16, University of Genoa, Italy.

10. Misale M, Bergles AE (1997) The influence of channel width on natural convection and boiling heat transfer from simulated microelctronic components. Exp Thermal Fluid Sci 14: 187-193.

11. Bonekamp S, Bier K (1997) Influence of ultrasound on pool boiling heat transfer to mixtures of the refrigerants R23 and R134a. Int J Refrigeration 20: 606-615.

12. Kenning DBR, Yan Y (1996) Pool boiling heat transfer on thin plate: features revealed by liquid crystal thermography. Int J Heat Mass Transfer 30: 3117-3137.

13. Bonjour J, Lallemand M (1998) Flow patterns during boiling in a narrow space between two vertical surfaces. Int J Multiphase Flow 24: 947-960.

14. Ammerman CN, You SM, Hong YS (1996) Identification of pool boiling heat transfer mechanisms from wire immersed in saturated FC-72 using a single-photo/LDA method. J Heat Transfer 118: 117-123. 